"This is the pre-peer reviewed version of the following article: Vladimir Sayevich, Bin Cai, Albrecht Benad, Danny Haubold, Luisa Sonntag, Nikolai Gaponik, Vladimir Lesnyak, and Alexander Eychmüller (2016). 3D Assembly of All-Inorganic Colloidal Nanocrystals into Gels and Aerogels. Communications (Weinheim an der Bergstrasse, Germany) Vol. 55 (21), pp. 6334-6338, which has been published in final form at DOI: 10.1002/anie.201600094.

This article may be used for non-commercial purposes in accordance with Wiley Terms and Conditions for Self-Archiving."

\title{
3D Assembly of All-Inorganic Colloidal Nanocrystals into Gels and Aerogels
}

\author{
Vladimir Sayevich, Bin Cai, Albrecht Benad, Danny Haubold, Luisa Sonntag, Nikolai Gaponik, Vladimir \\ Lesnyak*, and Alexander Eychmüller
}

\begin{abstract}
We report on an efficient assembly approach to a variety of electrostatically stabilized all-inorganic semiconductor nanocrystals (NCs) via their linking with appropriate ions into multibranched gel networks. These all-inorganic non-ordered 3D assemblies can combine strong interparticle coupling which facilitates charge transport between the NCs with their diverse morphology, composition, size, and functional capping ligands. Moreover, the resulting dry gels (aerogels) are highly porous monolithic structures, which preserve the quantum confinement of their building blocks. The inorganic semiconductor aerogel made of $4.5 \mathrm{~nm}$ CdSe colloidal NCs, capped with iodide ions and bridged with $\mathrm{Cd}^{2+}$ ions, exhibited a surface area as high as $146 \mathrm{~m}^{2} / \mathrm{g}$.
\end{abstract}

Colloidal semiconductor nanocrystals (NCs) attract stupendous scientific and technological interest owing to their size- and shape tunable optical, electrical and magnetic properties. ${ }^{[1]}$ Transferring these specific characteristics via their self-assembly in 3D superstructures will allow not only for bridging the macro- and nano-dimensions, but also for the creation of completely new properties as a result of their collective interparticle interactions. ${ }^{[2]}$ Gels and aerogels built from various colloidal NCs have recently been shown to provide this opportunity. ${ }^{[3]}$

Nowadays, the assembly of a variety of nanoparticles, in particular noble metal and semiconductor NCs, into functional non-ordered architectures is quite well developed and can easily be realized while maintaining the nanoscale properties. However, in many cases the resulting gel materials still have to be further processed in order to improve their properties relevant for desired practical applications. For instance, the surface capping of NCs is a critical aspect in the fabrication of functional gels, as it is mainly based on various organic molecules employed as stabilizing ligands in the colloidal synthesis of the nanoparticles. These

[*] V. Sayevich, B. Cai, A. Benad, D. Haubold, L. Sonntag, Prof. N. Gaponik, Dr. V. Lesnyak, Prof. A. Eychmüller

Physical Chemistry and Center for Advancing Electronics Dresden (cfAED)

TU Dresden

Bergstr. 66b, 01062 Dresden (Germany)

E-mail: vladimir.lesnyak@chemie.tu-dresden.de

Supporting information for this article is given via a link at the end of the document. ligands lead to an increase of the amount of organics in the gel structure and thus may hinder the accessibility of the active NC surface. The latter is of paramount importance in the application of these materials as catalysts. Further processing of the wet NCgels may cause the formation of multiple byproducts from organic ligands leading to unpredictable changes in the properties of the final material.

Alternatively, cheap and tiny inorganic ligands can efficiently replace the original organic molecules on the surface of the NCs thus providing electrostatic stabilization in polar solvents. These new ligands have a high affinity to the surface of the NCs, preserving their electronic structure and photophysical characteristics. ${ }^{[4]}$ The diversity of the inorganic species enables the modulation of a wide variety of properties, including the type of the conduction dominating carrier and its mobility, the photoluminescence $(\mathrm{PL})$, and the catalytic activity. ${ }^{[5]}$ Moreover, close-packed NC solids capped with appropriate inorganic ligands showed unprecedented electronic characteristics with strong coupling interactions, providing bright prospects for semiconductor technologies based on colloidal nanomaterials. ${ }^{[4 a}$ 6]

This motivated us to develop a novel approach to form 3D non-ordered assemblies based on NCs capped with inorganic ligands. We explored an extended material series of all-inorganic semiconductor NCs with different morphologies, compositions, sizes and capping ligands as building blocks for the formation of all-inorganic gels. In particular, we employed spherical NCs, nanorods and nanoplatelets. Previously our group developed strategies like the mild and well-controllable destabilization of NC colloids $^{[7]}$ and their linking via metal ion-assisted complexation ${ }^{[8]}$ to interconnect NCs via suitable ions leading to multibranched open networks. During the preparation of this paper, the Milliron group reported the first successful fabrication of an all-inorganic gel based on CdSe NCs capped with complex $\left[\mathrm{Ge}_{2} \mathrm{Se}_{6}\right]^{4-}$ ligands through their coordination with $\mathrm{Pt}^{2+}$ ions. ${ }^{[9]}$ However, this gelation approach may affect the optoelectronic characteristics of the NC building blocks. Additionally, an unintentional cation exchange with linking ions may also lead to undesirable modifications of the $\mathrm{NC}$ constituents in the gels.

In this work, we synthesized spherical colloidal CdSe, PbS, $\mathrm{PbSe}, \mathrm{ZnO}$ semiconductor NCs with narrow particle size distribution, as well as $\mathrm{CdSe}$ nanoplatelets and $\mathrm{ZnO}$ nanorods capped with native long chain, insulating organic ligands (details 
of all experiments are provided in the Supporting Information). Subsequently we completely displaced these molecules with different inorganic ligands, such as $\mathrm{S}^{2-}, \mathrm{I}^{-}, \mathrm{Cl}^{-}, \mathrm{F}^{-}, \mathrm{Ga}-\mathrm{I}-$ and $\mathrm{In}-$ $\mathrm{Cl}$-complexes. The nucleophilic nature and their high affinity to the surfaces of the NCs provide electrostatic stabilization for the colloidal dispersions in the polar solvent $N$-methylformamide (MFA) in a wide range of concentrations. Transmission electron microscopy (TEM) images of the inorganic-stabilized NCs confirmed the retention of their sizes, shapes and narrow size distributions after the ligand exchange (Figure SI1).
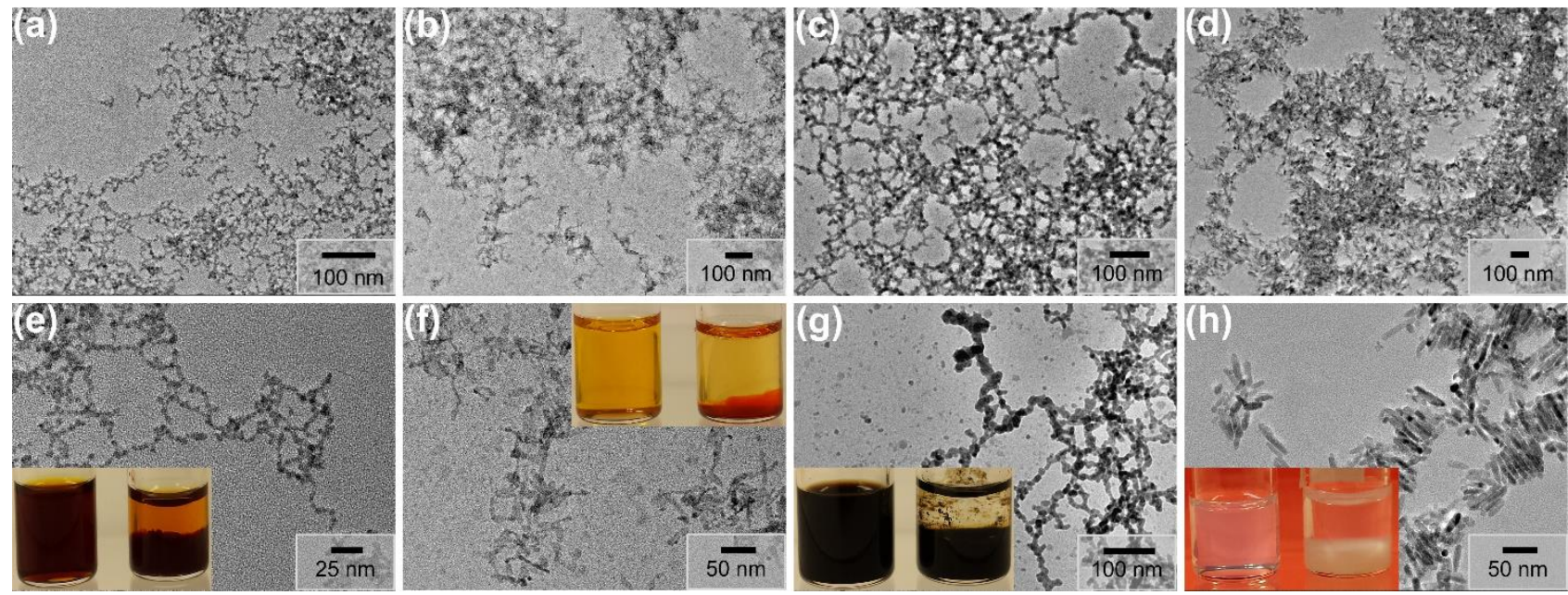

Figure 1. TEM images of a variety of all-inorganic NC gels formed using appropriate counterions: $\mathrm{CdSe}\left(\mathrm{I}^{-}\right) \mathrm{NCs} / \mathrm{Cd} \mathrm{d}^{2+}(\mathrm{a}, \mathrm{e}) ; \mathrm{CdSe}$ nanoplatelets $\left(\mathrm{I}^{-}\right) / \mathrm{Cd}{ }^{2+}(\mathrm{b}, \mathrm{f})$, $\mathrm{PbSe}\left(\mathrm{I}^{-}\right) \mathrm{NCs} / \mathrm{Pb}^{2+}(\mathrm{c}, \mathrm{g}) ; \mathrm{ZnO}$ nanorods $\left(\mathrm{F}^{-}\right) / \mathrm{Zn}^{2+}(\mathrm{d}, \mathrm{h})$. The insets are photographs of the corresponding NC dispersions before (left) and after (right) gelation.

In the solutions of the inorganically capped NCs the excitonic features in their optical absorption spectra were preserved (Figure SI2). The disappearance of the infrared absorption bands related to the characteristic $\mathrm{C}-\mathrm{H}$ stretching modes $\left(2700-3000 \mathrm{~cm}^{-1}\right)$ in inorganic-capped NCs confirmed the complete removal of the original organic ligands (Figure SI3), in agreement with our recent detailed studies of inorganic functionalization of various NCs. ${ }^{[4 c}$, 10] The successful functionalization of the NC surfaces with charged species was confirmed by electrophoretic measurements revealing characteristic values of the electrokinetic potentials $(\xi)$ of the particles which are related to their surface charge density (Figure SI4, Table SI1).

The next step was to assemble the NCs into 3D networks. To produce porous and multibranched gels, the choice of salts containing appropriate coordinating cations was of high importance. The Talapin group recently reported that $\mathrm{Cd}^{2+}$ ions showed the highest affinity among the variety of the studied cations to the surface of $\mathrm{S}^{2-}$-capped CdSe NCs leading to the highest surface charge inversion. ${ }^{[5]}$ We assumed that the chemical similarity of the ions in this process is likely to play a crucial role. That is why the gelation of the inorganicfunctionalized $\mathrm{CdSe}, \mathrm{PbSe}$, and $\mathrm{ZnO} \mathrm{NCs}$ was induced by adding $\mathrm{Cd}^{2+}, \mathrm{Pb}^{2+}, \mathrm{Zn}^{2+}$ ions, respectively, in the form of dehydrated acetate salts. The range of salts containing anions with low nucleophilicity can be extended to e.g. nitrates and perchlorates completely excluding organic residues in the resulting gels. Although these salts were successfully used to gelate the $\mathrm{CdSe}\left(\mathrm{I}^{-}\right) \mathrm{NCs}$, their different solubility in the chosen solvents or mixtures of solvents complicated the comparative studies of the gelation of different NCs (see the SI for more details). The possible versatility of this gelation approach can also be extended as was presented in our previous work by employing different cations for linking. ${ }^{[\mathrm{b}]}$

To induce a metal-ion assisted assembly of $\mathrm{CdSe}\left(\mathrm{I}^{-}\right) \mathrm{NCs}$ we added a solution of $0.2 \mathrm{M}$ of cadmium acetate to $3 \mathrm{mg} / \mathrm{mL} \mathrm{NC}$ dispersion in MFA which corresponded to a final concentration of $20 \mathrm{mM}$ of $\mathrm{Cd}^{2+}$ ions. Important to note that the gelation of other combinations of NCs with bridging ions was carried out in a similar way, but the efficiency of the conversion of the NCs into gels and the gelation time was varied due to some uncertainties in the determination of the NC concentrations, their different sizes, morphologies or surface charge density (Figure 1, Table SI2).

Both concentrations, that of the linking cation and that of the NC dispersion played an important role in determining the kinetics of the network formation and the structure of the resulting gel. Thus, the decrease of the concentrations of either the linking cations or the NCs or both did not result in any visible traces of destabilization of the system within months. On the contrary, the increase of the concentrations of $\mathrm{Cd}^{2+}$ and $\mathrm{CdSe}\left(\mathrm{I}^{-}\right) \mathrm{NCs}$ led to their immediate precipitation in the form of dense sediments. In turn, a balanced cation/NC ratio as well as their concentrations yielded multibranched structures within one week with a gel conversion efficiency of $\sim 80 \%$ (i.e. $80 \%$ of the initial NC content was transformed into the gel).

The advantage of using charged all-inorganic NCs as building blocks is their inclination to form gel networks via bridging by counterions, independent on their size, composition, morphology and even charge (Figure SI5). For example, $\mathrm{S}^{2-}$-capped CdSe NCs charge inverted with $\mathrm{Cd}^{2+}$ (i.e. positively charged, in our formulation $\left.\mathrm{CdSe}\left(\mathrm{S}^{2-}\right)\left(\mathrm{Cd}^{2+}\right)\right)$ may be gelated by adding $\mathrm{S}^{2-}$ ions acting as linkers. This can be explained as follows. The NCs may be considered as lyophobic colloids, which are thermodynamically metastable (with respect to the bulk solid) and 
rendered "stable" only in a kinetic sense. The kinetics of gelation (coagulation) will be mainly determined by overcoming the potential energy barrier providing the NC stability, i.e. their repulsion. After adding the linking agent, the charged cations adsorb on a few sites of the NC surface which is negatively charged, increasing the attractive potential contribution.
Additionally, to guarantee an effective bridging and the simultaneously occurring charge neutralization, there ought to be sufficient bare space on the surface of neighboring NCs to promote an effective adsorption of positively charged area during a particle approach, and preferably with enough space for more than one contact.

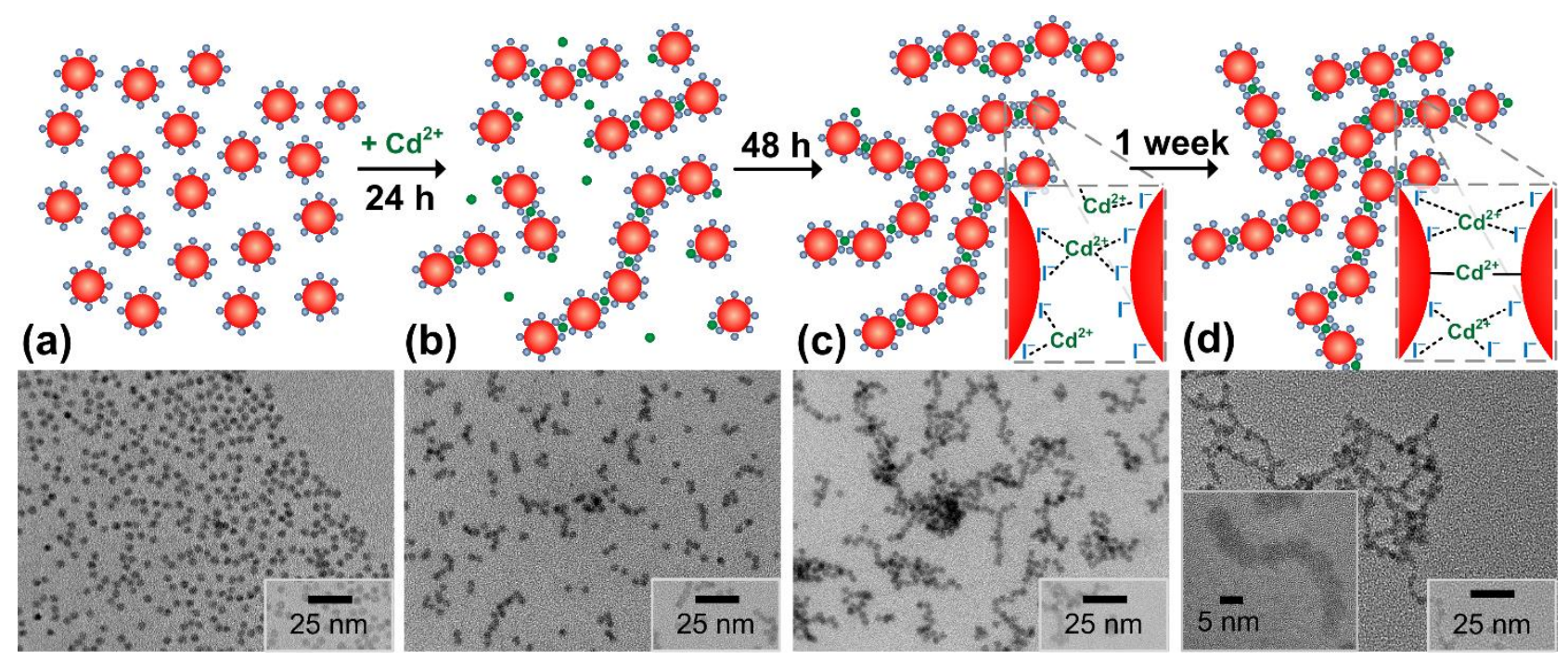

Figure 2. Scheme and associated TEM images showing the gelation of the $\mathrm{CdSe}\left(\mathrm{I}^{-}\right) \mathrm{NCs}(\mathrm{a})$ in $24 \mathrm{~h}(\mathrm{~b}), 48 \mathrm{~h}$, (c) and 1 week (d) after the addition of linking Cd+ ions.

When the concentration of the NCs is low, the probability of particle-particle encounters is also low, and with a high enough concentration of the counterions the reversion of the NC surface charge and the formation of a stable suspension may take place. Oppositely, at high concentrations of the ions and the NCs the kinetics of coagulation is fast yielding compact and dense aggregates via bridging through a larger average number of cations attached to each particle. ${ }^{[5]}$ Therefore, only an optimal concentration of NCs and counterions results in the formation of open porous structures. With the time of gelation, the local coordination environment of the bridging ions can vary across the NC network, accompanied with its reconstruction and strengthening of bonds between the structural units. This process is known as aging, which depends on the nature of the structural blocks and the ligands, their concentrations, surface charge densities, etc., and varies from sample to sample (Table SI2). The aging time is of high importance for the gel systems which, like ours, do not possess excellent complexing capabilities. We assume that during the aging, the formation of strong covalent bonds such as $\mathrm{I}-\mathrm{Cd}-\mathrm{I}$ and Se-Cd-Se takes place accompanied by interfusion of the NCs determining the rigid framework of the gel structure (Figure 2). The lack of reversibility upon using strong complexation agents (EDTA or its salts) additionally confirms the formation of strong bonds between the NCs. Moreover, other ions such as $\mathrm{Pb}^{2+}$ and $\mathrm{Zn}^{2+}$ were used to initiate the gelation of the $\mathrm{CdSe}\left(\mathrm{I}^{-}\right) \mathrm{NCs}$, albeit the kinetics and optical properties of the resulting gels differed from the Cd-linked particles. Since the colloidal stability of charged NCs is strongly determined by the concentration of the electrolyte (known as the Schulze-Hardy rule), we performed a control experiment by adding to the
$\mathrm{CdSe}\left(\mathrm{I}^{-}\right) \mathrm{NCs}$ a solution of $60 \mathrm{mM}$ of $\mathrm{NaOAc}$ with an ionic strength identical to $20 \mathrm{mM} \mathrm{Cd}(\mathrm{OAc})_{2}$. In this case we did not observe any aggregation of the NCs which confirms that their bridging is induced by linking via $\mathrm{Cd}^{2+}$ ions and not just due to an increased ionic strength of the solution. Nevertheless, we also consider the possibility of gel formation by altering the ionic strength, the dielectric constant, and the temperature as ways to change the magnitude of the surface charge density and the extent of the electrical double layer, i.e. induce gelation.

Furthermore, we monitored the aggregation of the $\mathrm{CdSe}\left(\mathrm{I}^{-}\right)$ NCs via TEM on early stages of the gelation. We observed a formation of chains (after 24 hours) with their subsequent branching and generation of more complex fractal aggregates (after 48 hours) which acted as building blocks to construct a wellconnected gel network (after 1 week) (Figure 2, Figure SI6). The NCs were randomly oriented in these gels, as revealed by high resolution TEM imaging (Figure 2d, Figure SI7). Further detailed studies will be needed to elucidate the gelation driving force with different contributions of the constituting components to the total interaction potential in this system using the Derjaguin-LandauVerwey-Overbeek (DLVO) theory, that was employed to describe the self-assembly of charged gold NCs. ${ }^{[11]}$ On the other hand, the involved changes in the excitonic features of seemingly identical solvogels based on CdSe NCs but capped with different inorganic ligands implies more complex interactions between the NCs, the capping ligands and the linkers in the assembled structure. These interactions may have not only electrostatic nature, as described by DLVO theory, and thus can lead to completely new functionalities (Figure SI2). 

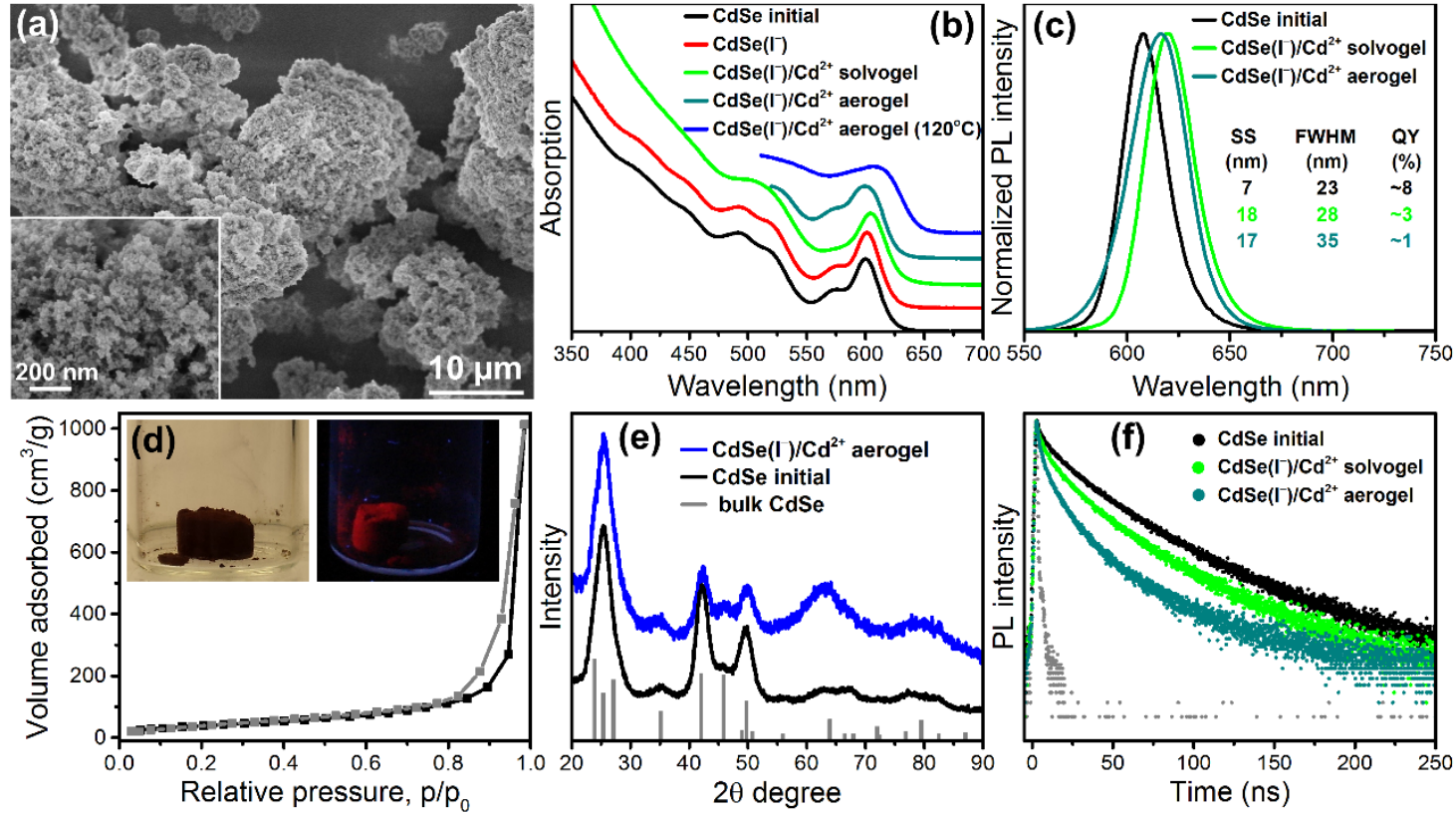

Figure 3. SEM image of an $\mathrm{I}^{-}$-capped CdSe NC aerogel linked with $\mathrm{Cd}^{2+}$ ions (a). Absorption spectra of the CdSe NCs before and after the ligand exchange with $\mathrm{I}^{-}$, their following gelation with $\mathrm{Cd}^{2+}$ in MFA and drying and heat treatment of the resulting aerogel (b). The spectra are vertically shifted for clarity. PL spectra of the initial CdSe NCs, $\mathrm{CdSe}\left(\mathrm{I}^{-}\right) / \mathrm{Cd}^{2+}$ wet gel and the aerogel with corresponding values of their Stokes shifts, FWHM, and PLQY (c). The nitrogen physisorption isotherms of the $\mathrm{CdSe}\left(\mathrm{I}^{-}\right) / \mathrm{Cd}^{2+}$ aerogel (d). The inset in (d) shows photographs of the CdSe aerogel monolith under day (left) and UV (right) light (PLQY 1\%). The XRD patterns of the initial CdSe NCs and the all-inorganic CdSe NC aerogel (e). The experimental pattern is compared to the database powder diffraction file of wurtzite CdSe (card \# 9011664). Time-resolved PL of the initial CdSe NCs, CdSe $\left(I^{-}\right) / \mathrm{Cd}^{2+}$ wet gel and the aerogel (f).

To prepare an aerogel from a CdSe NC wet gel, first MFA was replaced with acetone followed by the supercritical drying of the resulting solvogel according to a previously published procedure $^{[7 \mathrm{~b}]}$ to prevent the fine nanostructure from collapsing and to obtain the self-supporting aerogel monolith, whose scanning electron microscopy (SEM) image is displayed in Figure $3 a$. Figure $3 b, c, f$ shows the absorption, steady-state and timeresolved $\mathrm{PL}$ of the CdSe NCs before and after surface modification with $\mathrm{I}^{-}$, those of the $\mathrm{CdSe}\left(\mathrm{I}^{-}\right) / \mathrm{Cd}^{2+}$ solvogel in MFA, and those of the corresponding aerogel before and after activation in vacuum (at $120^{\circ} \mathrm{C}$ for 24 hours; this sample was further used for BET measurements).

In all cases we reveal the retention of excitonic features, accompanied by small shifts and broadening of the peaks, especially in the CdSe aerogel. The PL first was quenched after the ligand exchange with iodides and then partly restored in the solvogel, implying that $\mathrm{Cd}^{2+}$ ions can act both as a linker and as a passivating agent, suppressing non-radiative recombination introduced by the anions (quantum yield (QY) 3\%). At the same time, the PL maximum shifted to longer wavelengths $(18 \mathrm{~nm})$ likely due to a favorable exciton energy transfer from smaller (donor) to larger (acceptor) NCs. A slight shift to higher energies and a peak broadening accompanied by a PL drop (QY 1\%) occurred probably due to a partial surface etching, ligand desorption and increased size distribution after extensive washing. These changes in QYs are in agreement with timeresolved $\mathrm{PL}$ data. The decrease in the $\mathrm{PL}$ lifetime from the initial CdSe NCs to the solvogel and further to the aerogel is attributed to a formation of more channels for nonradiative deactivation through energy transfer and fast recombination on the nonpassivated surface states. The latter is especially pronounced in the aerogel. Heat treatment of the aerogel results in complete $\mathrm{PL}$ quenching and broadening of the first excitonic peak. This can be explained by a strengthening of the electronic coupling between the NCs via their partial interfusion, and by the influence of dipole-dipole interactions and (or) other factors related to the $\mathrm{NC}$ size distribution. Interestingly, the aerogels of the $\mathrm{CdSe}\left(\mathrm{I}^{-}\right)$ $\mathrm{NCs}$, linked with $\mathrm{Zn}^{2+}$ or $\mathrm{Pb}^{2+}$ ions showed optical properties differing from that one linked with $\mathrm{Cd}^{2+}$ with much lower QYs $(\sim 0.5 \%$ and $<0.1 \%$ vs. $3 \%$, respectively), confirming the importance of chemical similarity of the cations.

The increase of the cadmium content in the $\mathrm{CdSe}\left(\mathrm{I}^{-}\right) / \mathrm{Cd}^{2+}$ aerogel compared to the initial $\mathrm{CdSe}\left(\mathrm{I}^{-}\right) \mathrm{NCs}$ additionally confirms the linking role of $\mathrm{Cd}^{2+}$ in the formation of the network (Table SI3). Powder X-ray diffraction (XRD) analysis revealed no structural rearrangements in the gel compared to the parent CdSe NCs (Figure 3e). Thermogravimetric scans of the $4.5 \mathrm{~nm} \mathrm{CdSe}\left(\mathrm{I}^{-}\right) \mathrm{NCs}$ and the $\mathrm{Cd}^{2+}$-linked aerogel showed the total weight losses of $10 \%$ and $5 \%$, respectively, which were significantly smaller than that of $24 \%$ for the initial CdSe NCs (Figure SI8).

The resulting $\mathrm{CdSe}\left(\mathrm{I}^{-}\right) / \mathrm{Cd}^{2+}$ aerogel exhibited a remarkably large surface area of $146 \mathrm{~m}^{2} / \mathrm{g}$ as probed by nitrogen adsorptiondesorption via a BET analysis (Figure $3 \mathrm{~d}$ ). The isotherm resembles a type II curve with a sharp upturn in the high relative pressure region which is characteristic for CdSe aerogels as reported by the Brock group. ${ }^{[3 c]}$ This sharp upturn (lack of saturation) is indicative of liquid condensation associated with the presence of macropores. The SEM imaging of the CdSe aerogel unveiled the formation of a spongelike 3D structure.

The approach developed to form all-inorganic 3D assemblies based on a variety of NCs represents a powerful tool to tackle a crucial problem in the technological applications of NCs, namely 
how to build well-interconnected all-inorganic 3D structures while retaining the characteristic properties of the individual NCs. In the example of the $\mathrm{CdSe}\left(\mathrm{I}^{-}\right) / \mathrm{Cd}^{2+}$ aerogel we showed that the highly porous solid of chemically interconnected NCs still exhibited well pronounced features of the individual quantum dots. We further demonstrated that the proposed versatile gelation approach is applicable to a wide range of semiconductor NCs with different morphologies, compositions and sizes. This method will undoubtedly lead to materials for (opto)electronic applications, sensing, catalysis and thermoelectrics.

\section{Acknowledgements}

This work was supported by the Deutsche Forschungsgemeinschaft (DFG) within the Cluster of Excellence 'Center for Advancing Electronics Dresden' (cfAED) and by the European Research Council (ERC-2013-AdG project AEROCAT). We thank Nasser Mohamed-Noriega for the help in DLS measurements and Renate Schulze for ICP-OES measurements.

Keywords: semiconductor nanocrystals • inorganic ligands • interparticle linking $\bullet$ gels $\bullet$ aerogels

[1] a) C. B. Murray, C. R. Kagan, M. G. Bawendi, Annu. Rev. Mater. Sci. 2000, 30, 545; b) M. V. Kovalenko, L. Manna, A. Cabot, Z. Hens, D. V. Talapin, C. R. Kagan, V. I. Klimov, A. L. Rogach, P. Reiss, D. J. Milliron, P. Guyot-Sionnnest, G. Konstantatos, W. J. Parak, T. Hyeon, B. A Korgel, C. B. Murray, W. Heiss, ACS Nano 2015, 9, 1012; c) D. V Talapin, J.-S. Lee, M. V. Kovalenko, E. V. Shevchenko, Chem. Rev. 2010, 110, 389.

[2] J. J. Urban, D. V. Talapin, E. V. Shevchenko, C. R. Kagan, C. B. Murray, Nat Mater 2007, 6, 115.

[3] a) N. Gaponik, A.-K. Herrmann, A. Eychmüller, J. Phys. Chem. Lett. 2012, 8; b) J. L. Mohanan, I. U. Arachchige, S. L. Brock, Science 2005 307, 397; c) I. U. Arachchige, S. L. Brock, Acc. Chem. Res. 2007, 40, 801; d) W. Liu, A.-K. Herrmann, N. C. Bigall, P. Rodriguez, D. Wen, M. Oezaslan, T. J. Schmidt, N. Gaponik, A. Eychmüller, Acc. Chem. Res. 2015, 48, 154; e) C. Zhu, D. Du, A. Eychmüller, Y. Lin, Chem. Rev. 2015 115, 8896; f) S. Sánchez-Paradinas, D. Dorfs, S. Friebe, A. Freytag, A Wolf, N. C. Bigall, Adv. Mater. 2015, 27, 6152; g) A. Freytag, S. SánchezParadinas, S. Naskar, N. Wendt, M. Colombo, G. Pugliese, J. Poppe, C. Demirci, I. Kretschmer, D. W. Bahnemann, P. Behrens, N. C. Bigall, Angew. Chem.-Int. Edit. 2015, DOI: 10.1002/anie.201508972.

[4] a) M. V. Kovalenko, M. Scheele, D. V. Talapin, Science 2009, 324, 1417; b) A. Nag, M. V. Kovalenko, J.-S. Lee, W. Liu, B. Spokoyny, D. V. Talapin, J. Am. Chem. Soc. 2011, 133, 10612; c) V. Sayevich, N. Gaponik, M. Plötner, M. Kruszynska, T. Gemming, V. M. Dzhagan, S. Akhavan, D. R. T. Zahn, H. V. Demir, A. Eychmüller, Chem. Mater. 2015, 27, 4328; d) A. T. Fafarman, W.-k. Koh, B. T. Diroll, D. K. Kim, D.-K. Ko S. J. Oh, X. Ye, V. Doan-Nguyen, M. R. Crump, D. C. Reifsnyder, C. B. Murray, C. R. Kagan, J. Am. Chem. Soc. 2011, 133, 15753; e) S. J. Oh, N. E. Berry, J.-H. Choi, E. A. Gaulding, H. Lin, T. Paik, B. T. Diroll, S. Muramoto, C. B. Murray, C. R. Kagan, Nano Lett. 2014, 14, 1559.

[5] A. Nag, D. S. Chung, D. S. Dolzhnikov, N. M. Dimitrijevic, S Chattopadhyay, T. Shibata, D. V. Talapin, J. Am. Chem. Soc. 2012, 134, 13604.

[6] a) D. S. Dolzhnikov, H. Zhang, J. Jang, J. S. Son, M. G. Panthani, T. Shibata, S. Chattopadhyay, D. V. Talapin, Science 2015, 347, 425; b) J.H. Choi, A. T. Fafarman, S. J. Oh, D.-K. Ko, D. K. Kim, B. T. Diroll, S Muramoto, J. G. Gillen, C. B. Murray, C. R. Kagan, Nano Lett. 2012, 12, 2631.

[7] a) T. Hendel, V. Lesnyak, L. Kühn, A.-K. Herrmann, N. C. Bigall, L. Borchardt, S. Kaskel, N. Gaponik, A. Eychmüller, Adv. Funct. Mater.
2013, 23, 1903; b) N. Gaponik, A. Wolf, R. Marx, V. Lesnyak, K. Schilling, A. Eychmüller, Adv. Mater. 2008, 20, 4257.

[8] a) V. Lesnyak, S. V. Voitekhovich, P. N. Gaponik, N. Gaponik, A Eychmüller, ACS Nano 2010, 4, 4090; b) V. Lesnyak, A. Wolf, A Dubavik, L. Borchardt, S. V. Voitekhovich, N. Gaponik, S. Kaskel, A. Eychmüller, J. Am. Chem. Soc. 2011, 133, 13413; c) A. Wolf, V. Lesnyak N. Gaponik, A. Eychmüller, J. Phys. Chem. Lett. 2012, 3, 2188; d) S. V. Voitekhovich, V. Lesnyak, N. Gaponik, A. Eychmüller, Small 2015, 11, 5728.

[9] A. Singh, B. A. Lindquist, G. K. Ong, R. B. Jadrich, A. Singh, H. Ha, C. J. Ellison, T. M. Truskett, D. J. Milliron, Angew. Chem.-Int. Edit. 2015, 54, 14840.

[10] V. Sayevich, C. Guhrenz, M. Sin, V. M. Dzhagan, A. Weiz, D. Kasemann, E. Brunner, M. Ruck, D. R. T. Zahn, K. Leo, N. Gaponik, A. Eychmüller, Adv. Funct. Mater. 2016, DOI: 10.1002/adfm.201504767.

[11] H. Zhang, D. Wang, Angew. Chem.-Int. Edit. 2008, 47, 3984. 


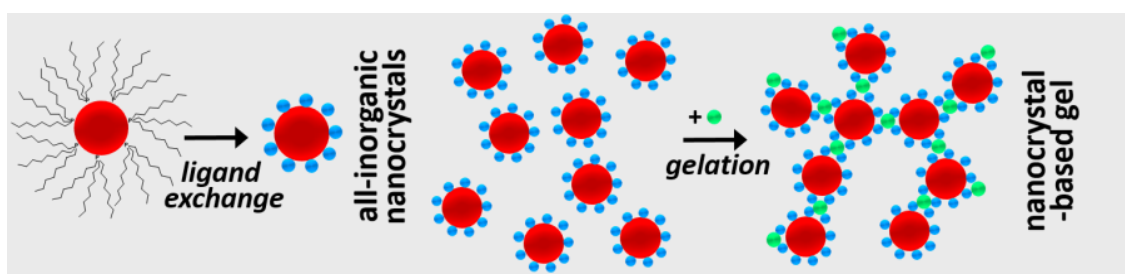

An efficient assembly approach for a variety of electrostatically stabilized allinorganic semiconductor nanocrystals via their linking with appropriate ions into multibranched gel networks was developed. The resulting aerogels are highly porous monolithic structures, which preserve the quantum confinement of their building blocks.
Vladimir Sayevich, Bin Cai, Albrecht Benad, Danny Haubold, Luisa Sonntag, Nikolai Gaponik, Vladimir Lesnyak, ${ }^{*}$ and Alexander Eychmüller

Page No. - Page No.

3D Assembly of All-Inorganic Colloidal Nanocrystals into Gels and Aerogels 